\title{
Total mercury concentrations in fish from Urrá reservoir (Sinú river, Colombia). Six years of monitoring
}

\author{
Concentraciones de mercurio total en peces del embalse Urrá (río \\ Sinú, Colombia). Seis años de monitoreo
}

\author{
José Marrugo-Negrete, ${ }^{1 *}$ Ph.D, Amado Navarro-Frómeta, ${ }^{2}$ Ph.D, Javier Ruiz-Guzmán, ${ }^{1}$ M.Sc.
}

\begin{abstract}
${ }^{1}$ University of Córdoba, Facult of Basic Sciences, Water Applied and Environmental Chemistry Group, Cra 6 \# 76-103, Monteria, Colombia. ${ }^{2}$ Technological University of Izúcar Matamoros. Prolongación Reforma \# 168, neighborhood Santiago Mihuacán, Izúcar of Matamoros, México. *Correspondence: jlmarrugon@hotmail.com
\end{abstract}

\begin{abstract}
Objective. The aim of this study was to monitor the total mercury $(\mathrm{T}-\mathrm{Hg})$ concentrations in fish from the Urrá reservoir, after impoundment. Materials and methods. Five fish species at different trophic levels were sampled from 2004 to 2009 and analyzed by cold-vapor atomic absorption spectroscopy for $\mathrm{T}-\mathrm{Hg}$ concentrations in muscle tissue. Water quality parameters were evaluated. Results. The highest $(1.39 \pm 0.69 \mu \mathrm{g} / \mathrm{g} \mathrm{ww})$ and lowest $(0.15 \pm 0.02 \mu \mathrm{g} / \mathrm{g} \mathrm{ww}) \mathrm{T}-\mathrm{Hg}$ concentrations were detected in Hoplias malabaricus (piscivorous) and Cyphocharax magdalenae (iliophagous/detritivorous) respectively, whereas Leporinus muyscorum (omnivorous) had an intermediate level $(0.40 \pm 0.11 \mu \mathrm{g} / \mathrm{g} \mathrm{ww})$. The organic matter content in the water increased with time and depth, whereas dissolved oxygen and $\mathrm{pH}$ decreased. A covariance analysis (with fish length as a covariate) shows a steady increase of T-Hg levels in all the studied species after impoundment. Conclusions. The T-Hg concentrations in the evaluated fish species, increased after impoundment. The water quality variables showed conditions favoring $\mathrm{Hg}$ methylation and its biomagnification, this last was evident in the fish food chain of the reservoir.
\end{abstract}

Received: September 2014; Accepted: February 2015.

Key words: Bioaccumulation, food chain, food habits (Source: $C A B$ ).

\section{RESUMEN}

Objetivo. El objetivo de este estudio fue monitorear las concentraciones de mercurio total ( $\mathrm{Hg}-\mathrm{T}$ ) en peces del embalse Urrá después del represamiento. Materiales y métodos. Cinco especies de peces de diferentes niveles tróficos fueron muestreadas de 2004 a 2009 y analizadas por espectroscopia de absorción atómica para las concentraciones de $\mathrm{Hg}-\mathrm{T}$ en tejido muscular. Se evaluaron parámetros de calidad de agua. Resultados. Las mayores $(1.39 \pm 0.69 \mu \mathrm{g} / \mathrm{g} \mathrm{ph})$ y menores $(0.15 \pm 0.02 \mu \mathrm{g} / \mathrm{g}$ ph) concentraciones de $\mathrm{Hg}-\mathrm{T}$ fueron detectadas en Hoplias malabaricus (piscívoro) y Cyphocharax magdalenae (iliófago/detritívoro) respectivamente, mientras que Leporinus muyscurum (omnívoro) tuvo un nivel intermedio $(0.40 \pm 0.11 \mu \mathrm{g} / \mathrm{g} \mathrm{ph})$. El contenido de materia orgánica en el agua se incrementó con el tiempo y la profundidad, mientras que el oxígeno disuelto y el pH disminuyeron. Un análisis de covarianza (con la longitud del pez como covariante) mostró un incremento sostenido de los niveles de $\mathrm{Hg}$-T en todas las especies evaluadas después del represamiento. Conclusiones. Las concentraciones de $\mathrm{Hg}$-T en las especies de peces evaluadas, incrementaron después del represamiento. Las variables de calidad de agua mostraron condiciones favorables para la metilación del $\mathrm{Hg}$ y su biomagnificación, estos último fue evidente en la cadena alimenticia de los peces del embalse.

Palabras clave: Bioacumulación, cadena alimenticia, hábitos alimenticios (Fuente: CAB). 


\section{INTRODUCTION}

Among the metals of environmental concern, mercury $(\mathrm{Hg})$ is considered a priority pollutant due to its ubiquity, persistency, accumulation, and toxicity. Monomethylmercury $(\mathrm{MeHg})$ is an important form of $\mathrm{Hg}$ because it is a potent neurotoxin with lipophilic characteristics and is able to bind proteins, causing severe damage to vertebrates (1). Hg uptake by humans occurs through the contaminated food ingestion and is the main route of uptake for populations that depend on fishing because $\mathrm{Hg}$ transferred to the biotic compartment is bioaccumulated and biomagnified by fish (2).

Reservoirs are created for various purposes, including hydropower generation, irrigation, flood control, water supply, fishery production, navigation and recreation. Although reservoirs provide significant benefits, they also have potential socioeconomic and environmental impacts (3). It has been demonstrated that the transfer of $\mathrm{Hg}$ to the aquatic food chain, increases in freshwater impoundments (4). Indeed, increased $\mathrm{Hg}$ levels in fish from hydroelectric reservoirs have been frequently reported, and these findings also show that fish species at high trophic levels have the highest $\mathrm{Hg}$ concentrations $(5,6)$.

The main source of $\mathrm{Hg}$ in reservoirs is the soil cover, which retains $\mathrm{Hg}$ bonded to organic matter. Other sources of $\mathrm{Hg}$, such as dry deposition and watershed runoff, may be important. The timing and magnitude of the response in fish to $\mathrm{Hg}$ concentrations will vary depending on ecosystem-specific variables such as sulfur and iron levels, $\mathrm{pH}$, the amount of flooded terrestrial carbon and $\mathrm{Hg}$ speciation (7). Given that the creation of hydroelectric reservoirs in the tropics generally involves the flooding of large areas of dense forest and the occurrence of high water temperatures, an increase in the activity of sulfate-reducing bacteria and plankton microorganisms in anoxic conditions, favored by the decomposition of submerged organic matter, also enhances $\mathrm{MeHg}$ production, primarily from sediments but also in the water column $(6,8)$.

In Colombia, there is evidence of $\mathrm{Hg}$ contamination in fish from sites traditionally dedicated to and/ or influenced by gold mining (9-11). However, although there are currently 24 active reservoirs in Colombia (12), with 10 more planned over the years $2010-2024$ (13), only there are published studies about the potential impact of dams on $\mathrm{Hg}$ concentrations in fish and the potential risk for human health resulting from continuing consumption of fish, for one reservoir $(14,15)$. In

\section{INTRODUCCIÓN}

Entre los metales de preocupación ambiental, el mercurio $(\mathrm{Hg})$ es considerado un contaminante prioritario debido a su ubicuidad, persistencia, acumulación y toxicidad. El Monometilmercurio ( $\mathrm{MeHg}$ ) es una forma importante de $\mathrm{Hg}$ debido a que es una potente neurotoxina con características lipofílicas y es capaz de unirse a las proteínas, causando daños severos a los vertebrados (1). La captación de $\mathrm{Hg}$ por los humanos ocurre a través de la ingesta de alimentos contaminados y es la principal ruta de exposición para poblaciones que dependen de la pesca debido a que el $\mathrm{Hg}$ transferido al compartimento biótico es bioacumulado y biomagnificado por los peces (2).

Los embalses son creados por varias razones, incluyendo generación de energía hidroeléctrica, irrigación, control de inundaciones, suministro de agua, producción pesquera, navegación y recreación. Aunque los embalses ofrecen beneficios significativos, también tienen potenciales impactos socioeconómicos y ambientales (3). Se ha demostrado que la transferencia de $\mathrm{Hg}$ a la cadena alimenticia acuática, incrementa en embalses de agua dulce (4). De hecho, niveles incrementados de $\mathrm{Hg}$ en peces de embalses hidroeléctricos son frecuentemente reportados, y estos hallazgos también muestran que las especies de peces de altos niveles tróficos tienen las mayores concentraciones de $\mathrm{Hg}(5,6)$.

La principal fuente de $\mathrm{Hg}$ en los embalses es la capa de suelo, que retiene $\mathrm{Hg}$ unido a la materia orgánica. Otras fuentes de $\mathrm{Hg}$, como la deposición seca y escorrentía de cuencas hidrográficas, pueden ser importantes. Los tiempos y la magnitud de la respuesta en los peces a las concentraciones de mercurio varían dependiendo de variables específicas del ecosistema tales como niveles de azufre y hierro, $\mathrm{pH}$, la cantidad de carbón terrestre inundado y la especiación de $\mathrm{Hg}$ (7). Dado que la creación de embalses hidroeléctricos en el trópico generalmente incluye la inundación de grandes áreas de bosque denso y la ocurrencia de altas temperaturas del agua, un incremento en la actividad de bacterias sulfatoreductoras y microorganismos del plancton en condiciones anóxicas, favorecida por la descomposición de la materia orgánica sumergida, también aumenta la producción de $\mathrm{MeHg}$, principalmente de los sedimentos pero también en la columna de agua $(6,8)$.

En Colombia, hay evidencia de contaminación de $\mathrm{Hg}$ en peces de sitios tradicionalmente dedicados a y/o influenciados por la minería de oro (9-11). Sin embargo, aunque existen actualmente 24 embalses activos en Colombia (12), con 10 más planeados entre los años 2010 y 2024 (13), sólo 
this regard, the aim of this study was to monitor the total mercury $(\mathrm{T}-\mathrm{Hg})$ concentrations in fish from the Urrá reservoir after impoundment.

\section{MATERIALS AND METHODS}

Study site. The Urrá hydroelectric power station is located on the Sinú River, in northwestern Colombia (N 7049'51" - 8०00'56", W 76¹2'45" $76^{\circ} 18^{\prime} 55^{\prime \prime}$ ) (Figure 1 ). The station is situated $30 \mathrm{~km}$ south of the municipality of Tierralta, Department of Córdoba, Colombia. The reservoir is fed by the Sinú River (average inflow $350 \mathrm{~m}^{3} / \mathrm{s}$ ) and its tributaries. Several of these tributaries are located in the Paramillo Nature Reserve catchment area, part of which was flooded in the year 2000 with the filling the reservoir. The flood area covers 7400 ha of tropical rain forest, with a monthly rainfall range from 20 to $350 \mathrm{~mm}$, with an annual average of $2212 \mathrm{~mm}$. A maximum temperature of 37.5 , a mean of 28.2 and a minimum of $18.7^{\circ} \mathrm{C}$ have been recorded $(16,17)$.

Sample collection and treatment. Five fish species with different food habits were investigated in this study. These species included the carnivorous-piscivorous Hoplias malabaricus (Moncholo) and Caquetaia kraussii (Mojarra amarilla), the omnivorous Leporinus muyscorum (Liseta) and the detritivoreiliophagous Prochilodus magdalenae (Bocachico) and Cyphocharax magdalenae (Yalúa). Specimens of each of the study species were caught during hay estudios publicados con relación al potencial impacto de los embalses en las concentraciones de $\mathrm{Hg}$ en peces y el riesgo potencial para la salud humana resultado del consumo continuo de pescado, para un embalse $(14,15)$. En este sentido, el objetivo de este estudio fue monitorear las concentraciones de mercurio total $(\mathrm{Hg}-\mathrm{T})$ en peces del embalse Urrá después del represamiento.

\section{MATERIALES Y MÉTODOS}

Sitio de estudio. La estación hidroeléctrica de Urrá está ubicada en el río Sinú, en el noreste de Colombia ( N 7049'51" - 8000'56", W $76^{\circ} 12^{\prime} 45^{\prime \prime}-76^{\circ} 18^{\prime} 55^{\prime \prime}$ ) (Figura 1). La estación está ubicada $30 \mathrm{~km}$ al sur del municipio de Tierralta, Departamento de Córdoba, Colombia. El embalse es alimentado por el río Sinú (caudal promedio de $350 \mathrm{~m}^{3} / \mathrm{s}$ ) y sus afluentes. Varios de estos afluentes están ubicados en las zonas de captación de la reserva natural Paramillo, una parte de la cual fue inundada en el año 2000 para llenar el embalse. El área de inundación cubre 7400 ha de bosque tropical húmedo, con una precipitación mensual entre 20 y 350 mm, con un promedio anual de $2212 \mathrm{~mm}$. Una temperatura máxima de $37.5^{\circ} \mathrm{C}$, media de $28.2^{\circ} \mathrm{C}$ y mínima de $18.7^{\circ} \mathrm{C}$ ha sido registrada en el área $(16,17)$. Recolección y tratamiento de muestras. En este estudio se investigaron cinco especies de peces con diferentes hábitos alimenticios. Esas especies incluyeron los carnívoros piscívoros Hoplias malabaricus (Moncholo) y Caquetaia kraussii (Mojarra amarilla), el

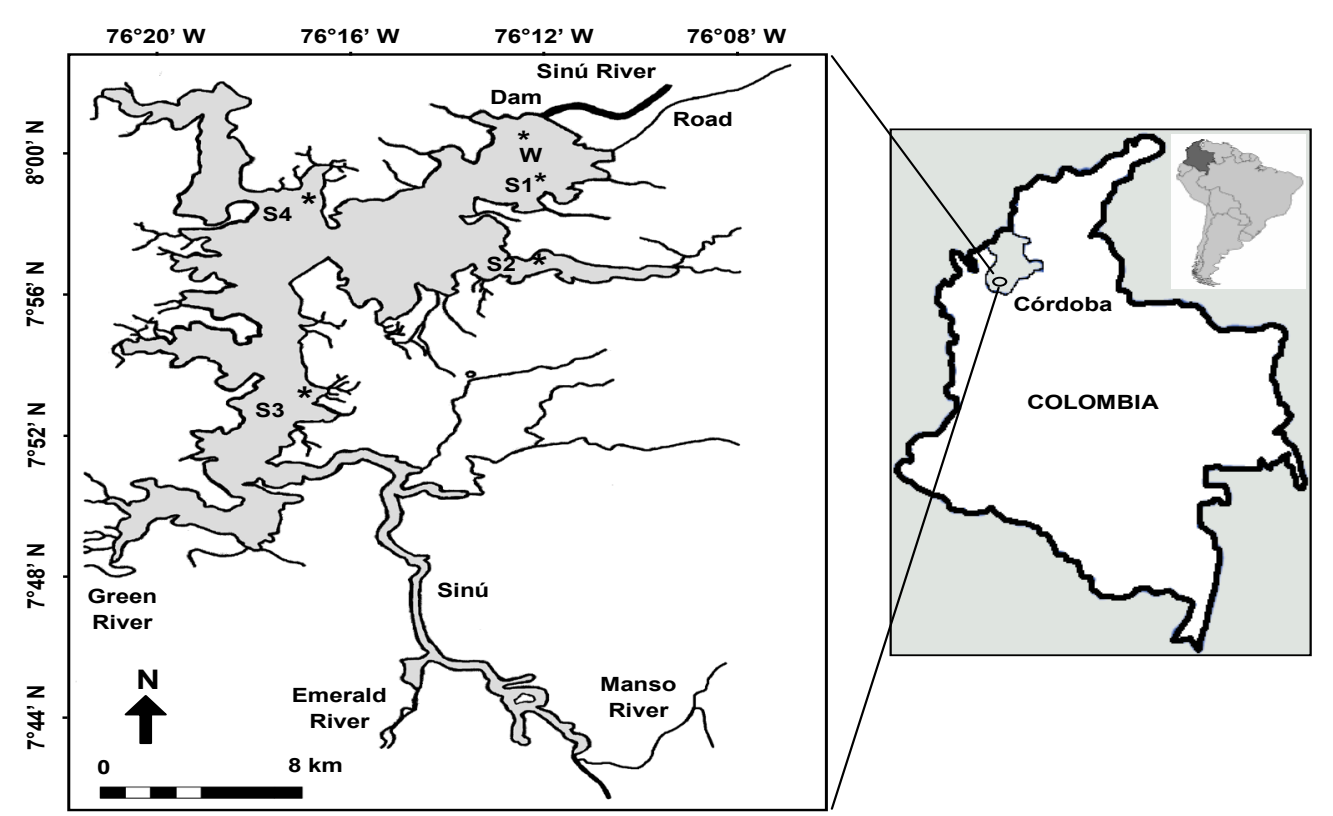

Figure 1. Study area: Urrá reservoir and sampling stations for water and sediments (S) and water (W). $\mathrm{S} 1=$ Chivogadó Village, S2=Km 40 Village, S3=Lourdes Stream, S4=Las Claras Stream. The fish samples were collected in the station S3 and S4. Adapted from Valderrama et al (16). 
six yearly sampling periods in January from 2004 to 2009, that is between four and nine years after filling the reservoir. The samples were collected with the help of local fishermen with a gill net. For each species, 3 - 6 samples were collected annually within the reservoir, 3 samples approximately $3 \mathrm{~km}$ downstream from the reservoir and 3 samples at a comparison site located approximately $60 \mathrm{~km}$ downstream from the reservoir. The fishes were individually packed, stored in iceboxes at $4^{\circ} \mathrm{C}$ and shipped to the laboratory, where they were immediately measured (standard length, from the nose to the caudal fin base: $( \pm 0.5 \mathrm{~cm})$ ). One piece of white muscle was removed from below the dorsal fin for the determination of $\mathrm{T}-\mathrm{Hg}$. The muscle samples were kept frozen at $-20^{\circ} \mathrm{C}$ until analysis.

From 2004 to 2008, water samples were collected at different depths $(0,5$ and $32 \mathrm{~m})$ using a Van Dorn bottle from a boat at one of the sites of maximum water depth of the reservoir (W station, Figure 1). The samples were used for the determination of water $\mathrm{pH}$, dissolved oxygen (DO) and total organic matter (OM). In 2009, water and sediment samples were collected at different stations on the reservoir ( $\mathrm{S}$ stations, Figure 1) for the analysis of $\mathrm{T}-\mathrm{Hg}$. The water samples ( $2 \mathrm{~L}$ per station) were collected with a polycarbonate Van Dorn bottle to $1 \mathrm{~m}$ below the surface of the water and then poured into clean, acid-washed polyethylene (HDPE) containers. The samples were acidified with $\mathrm{HNO}_{3}$ to $\mathrm{pH}<2$ and were kept refrigerated until analysis within 1 week after collection. Sediment samples were obtained by lowering a Van Veen grab from a boat. At each station, four sediment subsamples were collected at all cardinal points within a 2 $\mathrm{m}$ radius from the reference point. The samples were placed in plastic bags, labeled and packed in ice, transported to the laboratory, and dried at $40^{\circ} \mathrm{C}$ in a drying oven.

Mercury determination. The $\mathrm{T}-\mathrm{Hg}$ in the unfiltered water samples was measured using cold vapor atomic absorption spectroscopy (CVAAS) after digestion with a diluted $\mathrm{KMnO}_{4}{ }^{-}$ $\mathrm{K}_{2} \mathrm{~S}_{2} \mathrm{O}_{8}$ solution for $2 \mathrm{~h}$ at $95^{\circ} \mathrm{C}(18)$. The detection limit ( 3 times the standard deviation of 10 blank samples) was $0.1 \mu \mathrm{g} / \mathrm{L}$. The sediments were digested with $\mathrm{H}_{2} \mathrm{SO}_{4}-\mathrm{HNO}_{3}(7: 3, \mathrm{v} / \mathrm{v})$ and $\mathrm{KMnO}_{4}(5 \%, \mathrm{w} / \mathrm{v})$ at $100^{\circ} \mathrm{C}$ for $1 \mathrm{~h}(19)$, whereas the fish samples were treated with $\mathrm{H}_{2} \mathrm{SO}_{4}-\mathrm{HNO}_{3}$ $(2: 1, \mathrm{~V} / \mathrm{v})$ at $100-110^{\circ} \mathrm{C}$ for $3 \mathrm{~h}(14)$. The detection limits for the sediments and fish were $26.4 \mu \mathrm{g} / \mathrm{kg}$ dry weight ( $\mathrm{dw}$ ) and $13.1 \mu \mathrm{g} / \mathrm{kg}$ wet weight (ww), respectively. Quality control was performed with certified materials and spiked samples. The $\mathrm{T}-\mathrm{Hg}$ concentration for water (NIST, Standard Reference Material 1641d) was omnívoro Leporinus muyscorum (Liseta) y los detritívoros Prochilodus magdalenae (Bocachico) y Cyphocharax magdalenae (Yalúa). Especímenes de cada una de las especies del estudio fueron capturadas durante seis periodos de muestreo (uno por año) en enero de 2004 a 2009, esto es entre cuatro y nueve años después del llenado del embalse. Las muestras fueron recolectadas con la ayuda de pescadores locales con un trasmallo. Para cada especie, tres a seis muestras fueron recolectadas anualmente dentro del embalse, tres muestras aproximadamente $3 \mathrm{~km}$ río abajo del embalse y tres muestras en un sitio de comparación ubicado aproximadamente a $60 \mathrm{~km}$ río abajo del embalse. Los peces fueron individualmente empacados, almacenados en cajas con hielo a $4^{\circ} \mathrm{C}$ y enviados al laboratorio, donde fueron inmediatamente medidos (longitud estándar, desde la nariz hasta la base de la aleta caudal: $( \pm 0.5 \mathrm{~cm}))$. Un pedazo de músculo blanco fue removido de la parte inferior de la aleta dorsal para la determinación de $\mathrm{Hg}-\mathrm{T}$. Las muestras de músculo se mantuvieron congeladas a $-20^{\circ} \mathrm{C}$ hasta su análisis.

Entre 2004 y 2008, se recolectaron muestras de agua a diferentes profundidades $(0.5$ y 32 $\mathrm{m})$ utilizando una botella Van Dorn desde una embarcación en uno de los sitios de máxima profundidad del embalse (Estación W, Figura 1). Las muestras fueron utilizadas para determinar el $\mathrm{pH}$ del agua, oxígeno disuelto (OD) y materia orgánica total (MO). En 2009, se recolectaron muestras de agua y sedimentos en diferentes estaciones del embalse (Estaciones S, Figura 1) para el análisis de $\mathrm{Hg}-\mathrm{T}$. Las muestras de agua (2L por estación) fueron recolectadas con una botella de policarbonato Van Dorn a $1 \mathrm{~m}$ debajo de la superficie y luego vertidas en contenedores de polietileno (HDPE) limpios, lavados con ácido. Las muestras fueron acidificadas con $\mathrm{HNO}_{3}$ a $\mathrm{pH}<2$ y se mantuvieron refrigeradas hasta su análisis una semana después de su recolección. Las muestras de sedimentos se obtuvieron con una draga Van Veen desde una embarcación. En cada estación, cuatro sub muestras de sedimento fueron recolectadas en puntos cardinales en un radio de $2 \mathrm{~m}$ del punto de referencia. Las muestras fueron colocadas en bolsas plásticas, rotuladas y empacadas en hielo, transportadas al laboratorio y secadas a $40^{\circ} \mathrm{C}$ en un horno de secado.

Determinación de mercurio. $\mathrm{El} \mathrm{Hg}-\mathrm{T}$ en muestras sin filtrar de agua fue medido utilizando espectrometría de absorción atómica de vapor frío (CVAAS) luego de la digestión con una solución diluida de $\mathrm{KMnO}_{4}-\mathrm{K}_{2} \mathrm{~S}_{2} \mathrm{O}_{8}$ a $95^{\circ} \mathrm{C}$ (18). El límite de detección (tres veces la desviación estándar de 10 muestras testigo) fue $0.1 \mu \mathrm{g} / \mathrm{L}$. 
$1.581 \pm 0.022 \mathrm{mg} / \mathrm{L}$ (certified value, $1.590 \pm 0.018$ $\mathrm{mg} / \mathrm{L}$ ). The $\mathrm{T}-\mathrm{Hg}$ concentration for the biological material (DORM-2, dogfish muscle; National Research Council Canada) was $4.46 \pm 0.25 \mu \mathrm{g} / \mathrm{g}$ $\mathrm{dw}$ (certified value, $4.64 \pm 0.26 \mu \mathrm{g} / \mathrm{g} \mathrm{dw}$ ), and the concentration for sediments (CRM008-050; Resource Technology Corp.) was $0.74 \pm 0.02$ $\mu \mathrm{g} / \mathrm{g} \mathrm{dw}$ (certified value, $0.72 \pm 0.03 \mu \mathrm{g} / \mathrm{g} \mathrm{dw}$ ). The $\mathrm{Hg}$ recovery percentages from the spiked samples were $98.0 \pm 4.2 \%$ and $95.2 \pm 4.3 \%$ $(n=6)$ for sediments and fish, respectively. In both methods, the RSD was $<10 \%$. The $\mathrm{T}-\mathrm{Hg}$ concentrations in the sediments are reported on a dry weight basis, whereas those in fish are expressed on a fresh or wet weight basis. All mercury determinations were conducted in an S-Series 4 Atomic Absorption Spectrophotometer (Thermo Electron Corporation, United Kindom).

pH, DO and OM determination. $\mathrm{DO}$ and $\mathrm{pH}$ were measured in situ using a portable multimeter (Thermo Scientific, Orion 3STAR and Orion 4STAR models, respectively). The instrument for measuring dissolved oxygen was calibrated at the laboratory, and the $\mathrm{pH}$ meter was calibrated at each sampling site with two buffer solutions. The organic matter content was estimated based on standard methods of APHA (20).

Statistical treatment of data. To study the effects of site, species and year on $\mathrm{T}-\mathrm{Hg}$ concentrations, an analysis of variance (ANOVA) and an analysis of covariance (ANCOVA) were performed after checking the normality and homoscedasticity (Shapiro-Wilk and Bartlett tests) of the error term. The length of the fish was used as a covariate in the ANCOVA. If the null hypothesis (no factor effect) was rejected, a Duncan test was used to evaluate the differences between pairs of treatment means. For all statistical tests, $\mathrm{p}<0.05$ was considered significant. The slopes of the changes in $\mathrm{T}-\mathrm{Hg}$ with length for each species and year were determined by linear regression. The computations were performed with Statistica V 7.0 software.

\section{RESULTS}

T-Hg concentrations in fish. For all species, the $\mathrm{T}-\mathrm{Hg}$ levels in the fish collected in the reservoir were higher than were those at the comparison site during all sampling years (Table 1 ). In decreasing order of $\mathrm{T}-\mathrm{Hg}$ levels (and differences from the comparison site values), the species may be arranged in the order $H$. malabaricus $>$ C. kraussii $>$ L. muyscorum $>P$. magdalenae $\geq C$. magdalenae. The degree of contamination increased from detritivorous to piscivorous species, with omnivorous fish showing intermediate $\mathrm{T}-\mathrm{Hg}$ concentrations.
Los sedimentos fueron digeridos con $\mathrm{H}_{2} \mathrm{SO}_{4}-\mathrm{HNO}_{3}$ $(7: 3, \mathrm{v} / \mathrm{v})$ y $\mathrm{KMnO}_{4}(5 \%, \mathrm{p} / \mathrm{v})$ a $100^{\circ} \mathrm{C}$ por una hora (19), mientras que las muestras de peces fueron tratadas con $\mathrm{H}_{2} \mathrm{SO}_{4}-\mathrm{HNO}_{3}(2: 1, \mathrm{v} / \mathrm{v})$ a $100-110^{\circ} \mathrm{C}$ por tres horas (14). Los límites de detección para los sedimentos y los peces fueron $26.4 \mu \mathrm{g} / \mathrm{kg}$ peso seco (ps) y $13.1 \mu \mathrm{g} / \mathrm{kg}$ peso húmedo $(\mathrm{ph})$, respectivamente. El control de calidad se realizó con materiales certificados y muestras enriquecidas. La concentración de $\mathrm{Hg}-\mathrm{T}$ para el agua (NIST, Material Estándar de Referencia 1641d) fue $1.581 \pm 0.022 \mathrm{mg} / \mathrm{L}$ (valor certificado, $1.590 \pm 0.018 \mathrm{mg} / \mathrm{L}$ ). La concentración de $\mathrm{Hg}-\mathrm{T}$ para material biológico (DORM-2, músculo dogfish; Concejo Nacional de Investigación de Canadá) fue de $4.46 \pm 0.25 \mu \mathrm{g} / \mathrm{g}$ ps (valor certificado, $4.64 \pm 0.26 \mu \mathrm{g} / \mathrm{g} \mathrm{ps}$ ), y la concentración para sedimentos (CRM008-050; Resource Technology Corp.) fue de $0.74 \pm 0.02 \mu \mathrm{g} / \mathrm{g}$ ps (Valor certificado, $0.72 \pm 0.03 \mu \mathrm{g} / \mathrm{g} \mathrm{ps}$ ). Los porcentajes de recuperación de $\mathrm{Hg}$ para las muestras enriquecidas fueron de $98.0 \pm 4.2 \%$ y $95.2 \pm 4.3 \%(n=6)$ para sedimentos y peces, respectivamente. En ambos métodos, la desviación estándar relativa fue $<10 \%$. Las concentraciones de $\mathrm{Hg}$-T en los sedimentos se reportaron con base en su peso seco, mientras que en los peces se expresan con base en su peso freso o húmedo. Todas las determinaciones de $\mathrm{Hg}$ se realizaron con un Espectrómetro de Absorción Atómica S-Serie 4 (Thermo Electron Corporation, Reino Unido).

Determinación de pH, OD y MO. El pH y OD fueron medidos en el sitio utilizando un multímetro portátil (modelos Thermo Scientific, Orion 3STAR y Orion 4STAR, respectivamente). El instrumento para medir el oxígeno disuelto fue calibrado en el laboratorio, y el medidor de $\mathrm{pH}$ fue calibrado en cada sitio de muestreo con 2 soluciones buffer. El contenido de materia orgánica fue estimado con base en métodos estándar APHA (20).

Tratamiento estadístico de los datos. Para estudiar los efectos del sitio, especie y años sobre las concentraciones de $\mathrm{Hg}-\mathrm{T}$, se realizó un análisis de varianza (ANOVA) y un análisis de covarianza (ANCOVA) luego de verificar la normalidad y homosedasticidad (pruebas de Shapiro-Wilk y Bartlett) del término de error. La longitud del pez fue utilizada como covariable en el ANCOVA. Si la hipótesis nula (ningún efecto del factor) fue rechazad, una prueba de Duncan se utilizó para evaluar las diferencias entre pares de medias de tratamiento. Para todas las pruebas estadísticas, $p<0.05$ se consideró significativo. Las pendientes del cambio del $\mathrm{Hg}$-T con longitud para cada especie y años se determinaron por regresión linear. Los cómputos se realizaron con el software Statistica $\vee 7.0$ 
Table 1. $\mathrm{T}-\mathrm{Hg}$ concentrations $(\mu \mathrm{g} / \mathrm{g} w \mathrm{w})$ in fish from Urrá reservoir.

\begin{tabular}{|c|c|c|c|c|c|c|c|c|c|}
\hline \multirow{2}{*}{ Specie } & \multirow{2}{*}{$\mathbf{F H}^{\mathbf{a}}$} & \multirow{2}{*}{ Year } & \multicolumn{3}{|c|}{ Urrá Reservoir } & \multicolumn{3}{|c|}{ Comparison site } & \multirow{2}{*}{$\mathrm{UR} / \mathrm{CS}^{\mathrm{b}}$} \\
\hline & & & $n$ & Mean \pm SD & Interval & $n$ & Mean \pm SD & Interval & \\
\hline \multirow[t]{6}{*}{ H. malabaricus } & \multirow{6}{*}{ C } & 2004 & 9 & $0.626 \pm 0.192$ & $0.412-0.991$ & 3 & $0.125 \pm 0.025$ & $0.100-0.150$ & 5.0 \\
\hline & & 2005 & 10 & $0.814 \pm 0.537$ & $0.403-1.960$ & 3 & $0.121 \pm 0.035$ & $0.086-0.156$ & 6.7 \\
\hline & & 2006 & 10 & $1.380 \pm 0.759$ & $0.583-2.530$ & 3 & $0.128 \pm 0.048$ & $0.080-0.176$ & 10.8 \\
\hline & & 2007 & 10 & $1.388 \pm 0.687$ & $0.424-2.377$ & 3 & $0.149 \pm 0.051$ & $0.098-0.200$ & 9.3 \\
\hline & & 2008 & 10 & $1.339 \pm 0.601$ & $0.647-2.248$ & 3 & $0.161 \pm 0.102$ & $0.059-0.263$ & 8.3 \\
\hline & & 2009 & 8 & $1.324 \pm 0.430$ & $0.634-1.932$ & 3 & $0.161 \pm 0.044$ & $0.117-0.205$ & 8.2 \\
\hline \multirow[t]{5}{*}{ C. kraussii } & & 2004 & 6 & $0.450 \pm 0.087$ & $0.345-0.570$ & 3 & $0.115 \pm 0.028$ & $0.087-0.143$ & 3.9 \\
\hline & & 2005 & 6 & $0.562 \pm 0.144$ & $0.338-0.683$ & 3 & $0.131 \pm 0.018$ & $0.113-0.149$ & 4.3 \\
\hline & C & 2006 & 6 & $0.570 \pm 0.123$ & $0.432-0.730$ & 3 & $0.118 \pm 0.036$ & $0.082-0.154$ & 4.8 \\
\hline & & 2007 & 5 & $1.062 \pm 0.249$ & $0.738-1.405$ & 3 & $0.117 \pm 0.038$ & $0.079-0.155$ & 9.1 \\
\hline & & 2008 & 5 & $0.989 \pm 0.334$ & $0.688-1.385$ & 3 & $0.127 \pm 0.045$ & $0.082-0.172$ & 7.8 \\
\hline \multirow[t]{6}{*}{ L. muyscorum } & & 2004 & 10 & $0.282 \pm 0.060$ & $0.198-0.383$ & 3 & $0.084 \pm 0.018$ & $0.066-0.102$ & 3.4 \\
\hline & & 2005 & 10 & $0.344 \pm 0.057$ & $0.255-0.430$ & ns & ns & ns & - \\
\hline & & 2006 & 9 & $0.389 \pm 0.059$ & $0.320-0.498$ & ns & ns & ns & - \\
\hline & 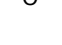 & 2007 & 8 & $0.405 \pm 0.111$ & $0.212-0.548$ & 3 & $0.077 \pm 0.023$ & $0.054-0.100$ & 5.3 \\
\hline & & 2008 & 8 & $0.376 \pm 0.082$ & $0.278-0.537$ & 3 & $0.082 \pm 0.034$ & $0.048-0.116$ & 4.6 \\
\hline & & 2009 & 9 & $0.362 \pm 0.075$ & $0.226-0.465$ & 3 & $0.098 \pm 0.019$ & $0.079-0.117$ & 3.7 \\
\hline \multirow[t]{6}{*}{ P. magdalenae } & & 2004 & 9 & $0.183 \pm 0.014$ & $0.163-0.208$ & 3 & $0.055 \pm 0.008$ & $0.047-0.063$ & 3.3 \\
\hline & & 2005 & 10 & $0.208 \pm 0.026$ & $0.178-0.267$ & 3 & $0.061 \pm 0.012$ & $0.049-0.073$ & 3.4 \\
\hline & T & 2006 & 9 & $0.221 \pm 0.026$ & $0.190-0.254$ & 3 & $0.052 \pm 0.008$ & $0.044-0.060$ & 4.3 \\
\hline & & 2007 & 10 & $0.233 \pm 0.035$ & $0.178-0.284$ & 3 & $0.052 \pm 0.011$ & $0.041-0.063$ & 4.5 \\
\hline & & 2008 & 7 & $0.203 \pm 0.028$ & $0.167-0.251$ & 3 & $0.060 \pm 0.009$ & $0.051-0.069$ & 3.4 \\
\hline & & 2009 & 9 & $0.206 \pm 0.034$ & $0.174-0.275$ & 3 & $0.063 \pm 0.017$ & $0.046-0.080$ & 3.3 \\
\hline \multirow[t]{5}{*}{ C. magdalenae } & & 2004 & 6 & $0.151 \pm 0.019$ & $0.128-0.174$ & 3 & $0.054 \pm 0.012$ & $0.042-0.066$ & 2.8 \\
\hline & & 2005 & 5 & $0.178 \pm 0.016$ & $0.161-0.197$ & ns & ns & ns & - \\
\hline & DI & 2006 & 4 & $0.180 \pm 0.012$ & $0.169-0.194$ & 3 & $0.057 \pm 0.009$ & $0.048-0.066$ & 3.2 \\
\hline & & 2007 & 4 & $0.207 \pm 0.019$ & $0.178-0.220$ & ns & ns & ns & - \\
\hline & & 2008 & 3 & $0.180 \pm 0.025$ & $0.156-0.206$ & 3 & $0.065 \pm 0.012$ & $0.053-0.077$ & 2.8 \\
\hline
\end{tabular}

a Food habit: $\mathrm{C}=$ Carnivorous, $\mathrm{O}=$ Omnivorous, $\mathrm{DI}=$ Detritivore iliophagous

batio $\mathrm{T}-\mathrm{Hg}$ in Urrá/T-Hg at comparison site; $\mathrm{ns}=$ no specimens caught

$\mathrm{T}-\mathrm{Hg}$ concentrations of fish from Urrá reservoir were normalized with the length for evaluate their evolution during the study period (Figure 2). The concentrations increased from 2004 to 2008 in the carnivorous fish, and from 2004 to 2007 in the omnivorous and detritivoruos fish. Subsequently, the $\mathrm{T}-\mathrm{Hg}$ levels showed a tendency to decrease.

Water parameters and $\mathbf{T}-\mathbf{H g}$ in fish. The changes in the water parameters from 2004 to 2008 showed an increase in the OM content

\section{RESULTADOS}

Concentraciones de $\mathrm{Hg}-\mathrm{T}$ en peces. Para todas las especies, los niveles de $\mathrm{Hg}-\mathrm{T}$ de los peces recolectados dentro del embalse fueron mayores que los del sitio de comparación durante todos los años de muestreo (Tabla 1). En orden decreciente de niveles de $\mathrm{Hg}-\mathrm{T}$ ( $\mathrm{y}$ con diferencias respecto al sitio de comparación), las especies pueden organizarse en el siguiente orden $H$. malabaricus $>$ C. kraussii $>$ L. muyscorum $>$ $P$. magdalenae $\geq C$. magdalenae. El grado de 


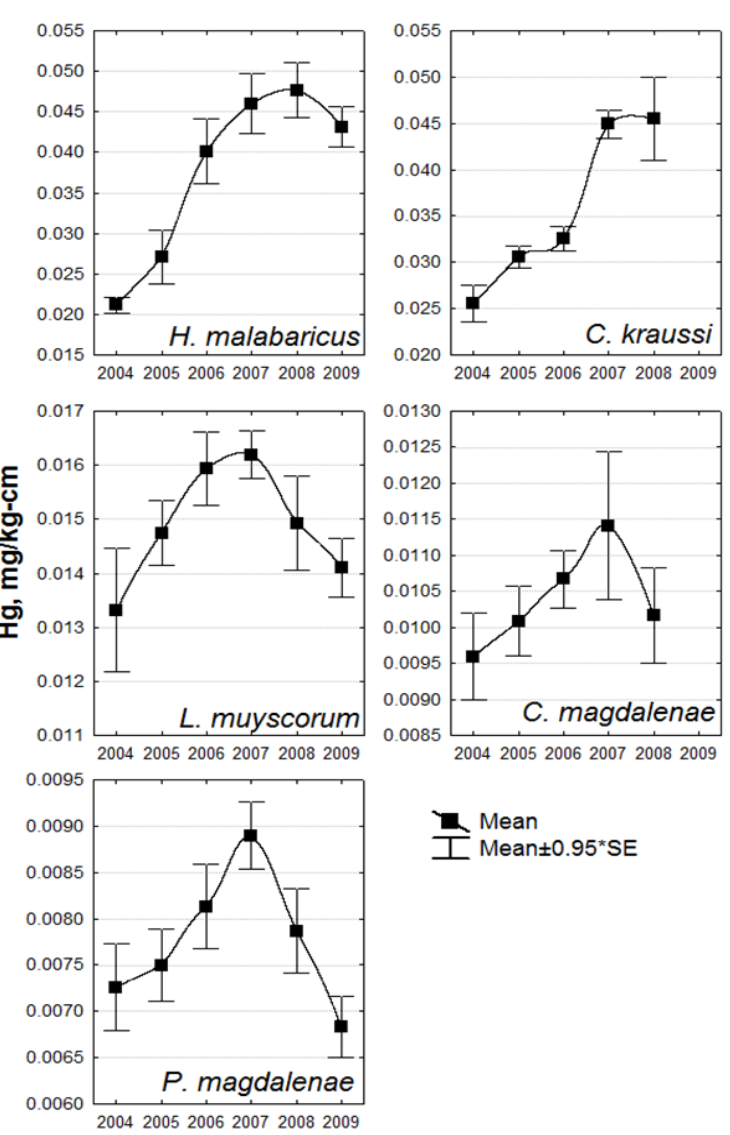

Figure 2. Values of T-Hg concentrations in fish from the Urrá reservoir normalized to unit length $(\mathrm{cm})$ vs year.

and decrease in $\mathrm{DO}$ and $\mathrm{pH}$ with time and depth (Figure 3 ). All variables were strongly and significantly correlated $(\mathrm{R} \geq 0.991)$. The $\mathrm{T}-\mathrm{Hg}$ concentrations of samples collected in 2009 were $173.2 \pm 14.1 \mu \mathrm{g} / \mathrm{kg} \mathrm{dw}$ in sediment and $0.14 \pm$ $0.04 \mu \mathrm{g} / \mathrm{L}$ in water. The analysis of correlation between $\mathrm{T}-\mathrm{Hg}$ concentration of fish with $\mathrm{OM}, \mathrm{pH}$

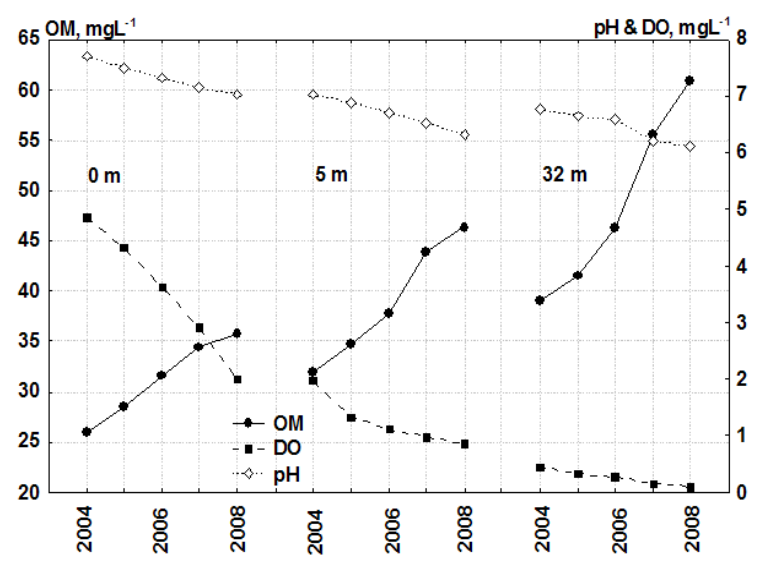

Figure 3. Measured water parameters from 2004 to 2008 in the Urrá reservoir at different depths $(0,5 \mathrm{y}$ $32 \mathrm{~m}) . \mathrm{OM}=$ Organic matter, $\mathrm{DO}=$ Dissolved oxygen. contaminación incrementó de especies detritívora a piscívora, con los peces omnívoros mostrando concentraciones intermedias de $\mathrm{Hg}-\mathrm{T}$.

Las concentraciones de $\mathrm{Hg}-\mathrm{T}$ de los peces del ensamble Urrá fueron normalizadas con la longitud para evaluar su evolución durante el periodo de estudio (Figura 2). Las concentraciones incrementaron entre 2004 y 2008 en los peces carnívoros, y entre 2004 y 2007 en los peces omnívoros y detritívoros. Subsiguientemente, los niveles de $\mathrm{Hg}-\mathrm{T}$ mostrar una tendencia a disminuir.

Parámetros del agua y Hg-T en peces. Los cambios en los parámetros de agua entre 2004 y 2008 mostraron un incremento del contenido de $\mathrm{MO}$ y una disminución del OD y $\mathrm{pH}$ con el tiempo y la profundidad (Figura $3)$. Todas las variables fueron fuertemente y significativamente correlacionadas $(R \geq 0.991)$. Las concentraciones de $\mathrm{Hg}-\mathrm{T}$ de las muestras recolectadas en 2009 fueron $173.2 \pm 14.1 \mu \mathrm{g} / \mathrm{kg}$ ps en sedimento y $0.14 \pm 0.04 \mu \mathrm{g} / \mathrm{kg}$ en agua. El análisis de correlación entre la concentración de $\mathrm{Hg}$ - $\mathrm{T}$ en peces con los valores de MO, OD y pH del agua, se presentan en la tabla 2 . Con base en los valores medios anuales, las concentraciones de $\mathrm{Hg}$-T para $C$. Kraussii fueron positiva y significativamente $(p<0.05)$ correlacionadas con la MO y negativamente correlacionadas con el OD y $\mathrm{pH}$. Para las otras especies, aunque las correlaciones no fueron significativas $(p>0.05)$, siguieron el mismo patrón.

Hg-T y longitud de los peces. Un modelo lineal ajustado de la concentración de $\mathrm{Hg}-\mathrm{T}$ como función de la longitud de los peces (Tabla 3) mostró una correlación significativa $(p<0.05)$ entre estas variables para los peces piscívoros en todos los años analizados y al menos en un año para las especies omnívoras y detritívoras. Los peces piscívoros mostraron las pendientes más agudas, seguidos por los peces omnívoros y detritívoros. Las pendientes incrementaron durante el periodo de estudio para todas las especies.

Los resultados del ANCOVA (modelo de pendientes separadas, con la longitud como covariable) se presentan en la tabla 4. Los resultados confirman en general la dependencia de las concentraciones de $\mathrm{Hg}$ - $\mathrm{T}$, de los hábitos dietarios de los peces y el año. Se encontraron excepciones a este patrón, luego de remover la influencia de la longitud, de especies omnívoras y detritívoras, donde marcados descensos en las concentraciones de $\mathrm{Hg}$-T después del 2007 están ausentes. C. Kraussii mostró un incremento sostenido en los niveles de $\mathrm{Hg}$ a lo largo del periodo de estudio, alcanzando valores mayores que los de $H$. malabaricus en 2007 y 2008. 
and DO values of water, is presented in table 2. Based on the annual mean values, the $\mathrm{T}-\mathrm{Hg}$ concentrations for $C$. kraussii was positive and significantly $(p<0.05)$ correlated with OM and negatively correlated with $\mathrm{DO}$ and $\mathrm{pH}$. For other species, although the correlations were not significant $(p>0.05)$, they followed the same pattern.

T-Hg and fish length. A fitted linear model

Table 2. Correlation coefficients of $\mathrm{T}-\mathrm{Hg}$ concentrations of fish with organic matter (OM), dissolved oxygen (DO) and $\mathrm{pH}$ of water.

\begin{tabular}{lccc}
\hline \multicolumn{1}{c}{ Specie } & OM & DO & pH \\
\hline H. malabaricus a & 0.8410 & -0.8729 & -0.8504 \\
C. kraussii & $\mathbf{0 . 9 4 0 8}$ & $\mathbf{- 0 . 8 8 6 2}$ & $\mathbf{- 0 . 9 2 6 1}$ \\
L. muyscorum & 0.7666 & -0.8185 & -0.7885 \\
P. magdalenae & 0.5139 & -0.5525 & -0.5309 \\
C. magdalenae & 0.6910 & -0.6983 & -0.7006
\end{tabular}

Significant correlations at $p<0.05$ in bold; a significant at $p<0.074$

of the $\mathrm{T}-\mathrm{Hg}$ concentration as a function of fish length (Table 3) showed a significant correlation $(p<0.05)$ between these variables for the piscivorous fish in all years analyzed and at least one year for omnivorous and detritivorous species. The piscivorous fish showed the steepest slopes, followed by the omnivorous fish and detritivorous fish. The slopes increased during the study period for all species.

The ANCOVA results (separate-slopes model, length - covariate) is presented in table 4 . The results generally confirm the observed dependence of $\mathrm{T}-\mathrm{Hg}$ concentrations on fish dietary habits and year. Exceptions to this pattern were
Table 5. Comparison of T-Hg levels found in fish in this study (in bold) with values from other studies in Colombia (Col) and Brazil (Br).

\begin{tabular}{|c|c|c|c|c|}
\hline Species/Diet ${ }^{\mathrm{a}}$ & $\begin{array}{l}\text { Trophic } \\
\text { Level }^{\mathrm{b}}\end{array}$ & Location $T$ & $\mathrm{~T}-\mathrm{Hg}(\mu \mathrm{g} / \mathrm{g} w w)$ & AL $^{9}$ \\
\hline \multirow[t]{4}{*}{ H. malabaricus/CP } & \multirow{4}{*}{4.5} & & $0.40-2.53$ & 29 \\
\hline & & Mojana, Colc & $0.11-0.67$ & 23 \\
\hline & & Ayapel, Cold & $0.12-0.58$ & 22 \\
\hline & & Mojana, Cole & $0.21-0.43$ & 28 \\
\hline \multirow[t]{2}{*}{ P. surinamensis/C } & \multirow[b]{2}{*}{4.5} & Ayapel, Cold & $0.12-0.65$ & 26 \\
\hline & & Mojana, Colc & $0.14-0.44$ & 24 \\
\hline P. fasciatum/C & 4.38 & L. Manso, $\mathrm{Br}^{\mathrm{f}}$ & $1.20-1.27$ & 81 \\
\hline S. $\operatorname{lima} / C$ & 4.1 & Mojana, Cole & $0.20-0.51$ & 35 \\
\hline \multirow[t]{2}{*}{ A. caucanus/C } & \multirow{2}{*}{3.86} & Mojana, Colc & $.27-1.00$ & 28 \\
\hline & & Mojana, Cole & $0.23-0.54$ & 25 \\
\hline S. brasiliensis/P & 3.79 & L. Manso, $\mathrm{Br}^{\mathrm{f}}$ & $0.50-1.62$ & 80 \\
\hline $\begin{array}{l}\text { S. spilopleura and } \\
\text { S. marginatus/C }\end{array}$ & $3.16 / 3.55$ & $\begin{array}{c}\text { L. Manso, } \\
\mathrm{Br}^{\mathrm{f}} \text {. }\end{array}$ & $0.29-2.00$ & 14 \\
\hline \multirow[t]{2}{*}{ C. kraussii/CP } & 3.36 & & 1.41 & 20 \\
\hline & & Mojana, Cole & $0.02-0.56$ & 17 \\
\hline \multirow[t]{3}{*}{ L. muyscorum/O } & & & $0.20-0.55$ & 24 \\
\hline & 2.1 & Mojana, Colc & $0.07-0.58$ & 22 \\
\hline & & Ayapel, Cold & $0.10-0.48$ & 24 \\
\hline B. hilarii & 2 & L. Manso, $\mathrm{Br}^{\mathrm{f}}$ & $0.07-1.03$ & 40 \\
\hline \multirow[t]{4}{*}{ P. magdalenae/D } & & & $0.16-0.28$ & 28 \\
\hline & 2.07 & Mojana, Colc & $0.04-0.22$ & 22 \\
\hline & & Ayapel, Cold & $0.04-0.23$ & 24 \\
\hline & & Mojana, Cole & $0.01-0.17$ & 18 \\
\hline C. mag & 2 & & $0.13-0.22$ & 22 \\
\hline
\end{tabular}

a $\mathrm{C}=$ Carnivorous, $\mathrm{P}=$ Piscivorous, $\mathrm{O}=$ Omnivorous, $\mathrm{D}=$ Detritivorous ; b Data from Fishbase (21); ${ }^{c}=(9) ; \mathbf{d}=(10) ;{ }^{\mathbf{e}}=(11) ;{ }^{\mathbf{f}}=(6) ;{ }^{\mathbf{g}}=$ Average length, $\mathrm{cm}$.

Table 3. Results of linear fitting of $\mathrm{T}-\mathrm{Hg}$ concentrations vs length of fish.

\begin{tabular}{|c|c|c|c|c|c|c|c|c|}
\hline \multirow{2}{*}{ Specie } & \multirow{2}{*}{$\mathbf{F H}$} & \multirow{2}{*}{ Parameter } & \multicolumn{6}{|c|}{ Year } \\
\hline & & & 2004 & 2005 & 2006 & 2007 & 2008 & 2009 \\
\hline \multirow{2}{*}{ H. malabaricus } & \multirow{2}{*}{$\mathrm{C}$} & slope & 0.0227 & 0.0590 & 0.0896 & 0.0851 & 0.0906 & 0.0667 \\
\hline & & $\mathrm{R}$ & 0.9011 & 0.8429 & 0.9469 & 0.9839 & 0.9455 & 0.9488 \\
\hline \multirow{2}{*}{ C. kraussii } & \multirow{2}{*}{ C } & slope & 0.0150 & 0.0224 & 0.0240 & 0.0609 & 0.1136 & ns \\
\hline & & $\mathrm{R}$ & 0.9439 & 0.9772 & 0.9807 & 0.9638 & 0.8848 & ns \\
\hline \multirow{2}{*}{ P. magdalenae } & \multirow{2}{*}{ DI } & slope & 0.0068 & 0.0081 & 0.0097 & 0.0185 & 0.0129 & 0.0253 \\
\hline & & $\mathrm{R}$ & 0.7858 & 0.9200 & 0.8592 & 0.9754 & 0.5187 & 0.9338 \\
\hline \multirow{2}{*}{ L. muyscorum } & \multirow{2}{*}{$\mathrm{O}$} & slope & 0.0010 & 0.0015 & 0.0021 & 0.0061 & 0.0022 & 0.0044 \\
\hline & & $\mathrm{R}$ & 0.4329 & 0.2802 & 0.3775 & 0.6851 & 0.1977 & 0.3961 \\
\hline \multirow{2}{*}{ C. magdalenae } & \multirow{2}{*}{ DI } & slope & 0.0038 & 0.0043 & 0.0046 & 0.0038 & 0.0059 & ns \\
\hline & & $\mathrm{R}$ & 0.9353 & 0.9379 & 0.6721 & 0.9488 & 0.9642 & ns \\
\hline
\end{tabular}

a Food habit: $\mathrm{C}=$ Carnivorous, $\mathrm{O}=$ Omnivorous, $\mathrm{DI}=$ Detritivore iliophagous

Note: Significant $(p<0.05)$ sum of squares of whole model/sum of squares of residuals are indicated in bold. ns $=$ no specimens caught 
Table 4. ANCOVA T-Hg least squares means, $\mu \mathrm{g} / \mathrm{g}^{\mathrm{a}}$.

\begin{tabular}{ccccccc}
\hline & \multicolumn{6}{c}{ Year } \\
\cline { 2 - 7 } Species & $\mathbf{2 0 0 4}$ & $\mathbf{2 0 0 5}$ & $\mathbf{2 0 0 6}$ & $\mathbf{2 0 0 7}$ & $\mathbf{2 0 0 8}$ & $\mathbf{2 0 0 9}$ \\
\hline H. malabaricus & 0.524 & 0.635 & 0.746 & 1.116 & 1.171 & 0.997 \\
C. kraussii & 0.553 & 0.702 & 0.746 & 1.165 & 1.418 & $\mathrm{~ns}$ \\
L. muyscorum & 0.301 & 0.353 & 0.392 & 0.412 & 0.375 & 0.354 \\
P. magdalenae & 0.182 & 0.203 & 0.215 & 0.226 & 0.201 & 0.183 \\
C. magdalenae & 0.185 & 0.209 & 0.217 & 0.231 & 0.222 & $\mathrm{~ns}$ \\
\hline
\end{tabular}

a Computed for covariate at its means.

Current effect: $F(18,159)=2.2406, p=0.00424 . n s=$ no specimens caught

founded, after the removal of length influence, for omnivorous and detritivorous species, where sharp decreases in $\mathrm{T}-\mathrm{Hg}$ concentrations after 2007 are absent. C. kraussii showed a steady increase in $\mathrm{Hg}$ levels throughout the study period, reaching values higher than $H$. malabaricus in 2007 and 2008.

\section{DISCUSSION}

The higher $\mathrm{T}-\mathrm{Hg}$ concentrations of fish from the Urrá reservoir than in the comparison site (Table 1 ) show the $\mathrm{Hg}$ accumulation in reservoir, and their increase with the length of fish (Table 3 ) suggest an $\mathrm{Hg}$ biomagnification process within the aquatic food web. These results are remarkable because the levels of $\mathrm{T}-\mathrm{Hg}$ detected in the fish samples of Urrá reservoir, are generally higher for the same trophic level than those reported in other regions of Colombia and Brazil in the presence of strong mining activity (Table 5). Note that the carnivorous and piscivorous fish investigated in Lago Manso, Brazil, had on average, twice the length of those evaluated in the present study.

Is important to note that average $\mathrm{T}-\mathrm{Hg}$ concentration of $H$. malabaricus in the years 2006 to 2009 and C. kraussii in the years 2007 and 2008, exceeded the guideline level for carnivorous fish $(1 \mu \mathrm{g} / \mathrm{g}$ ) suggested in the codex alimentarius for human consumption (22). The addition of factors as the high $\mathrm{T}-\mathrm{Hg}$ concentration, the high fish consumption reported in the study zone [148 g/day in 2005 (16) and $76.3-228.8 \mathrm{~g} /$ day in $2010(15)$ ], the fact that $H$. malabaricus represented the $32.2 \%$ of fish total catch in the reservoir in 2005 (16) and the first and second place in the fish consumption into diet of children and women of childbearing age of the riverside population from Urrá reservoir in 2010 (15), suggest an increased risk for mercury exposure, which must be studied promptly.

Although there are no punctual sources of $\mathrm{Hg}$ in the study area, a diffuse source exists in a nearby watershed (San Jorge River basin), where ferronickel, coal and gold mining occur. This activity has expanded

\section{DISCUSIÓN}

Las mayores concentraciones de $\mathrm{Hg}-\mathrm{T}$ de los peces del embalse Urrá que las del sitio de comparación (Tabla 1) muestran la acumulación de $\mathrm{Hg}$ en el embalse, y su incremento con la longitud del pez (Tabla 3) sugiere un proceso de biomagnificación de $\mathrm{Hg}$ dentro de la red alimenticia acuática. Éstos resultados son importantes porque los niveles de $\mathrm{Hg}$ - $\mathrm{T}$ detectados en las muestras de peces del embalse Urrá, son generalmente mayores para el mismo nivel trófico que las reportadas en otras regiones de Colombia y Brasil en presencia de fuerte actividad minera (Tabla 5). Se debe notar que los peces carnívoros y piscívoros investigados en Lago Manso, Brasil, tenían en promedio, el doble de longitud que los evaluados en el presente estudio.

Es importante tener en cuenta que la concentración promedio de $\mathrm{Hg}-\mathrm{T}$ en $\mathrm{H}$. malabaricus en los años 2006 a 2009 y C. Kraussii en los años 2007 y 2008, excedió el nivel de referencia en peces carnívoros para consumo humano $(1 \mu \mathrm{g} / \mathrm{g})$ sugerido en el Códex alimentarius (22). La adición de factor como alta concentración de $\mathrm{Hg}-\mathrm{T}$, el alto consumo de peces reportado en la zona de estudio (148 g/día en 2005 (16) y 76.3 - $228.8 \mathrm{~g} /$ día en 2010), el hecho que $H$. malabaricus representó un $32.2 \%$ de la captura total del ensamble en 2005 (16) y el primer y segundo lugar en el consumo de peces en la dieta de niños y mujeres en edad fértil de la población ribereña del embalse Urrá en 2010 (15), sugiere un riesgo incrementado de exposición a mercurio, que debe ser estudiado prontamente.

Aunque no hay fuentes puntuales de $\mathrm{Hg}$ en el área de estudio, una fuente difusa existe en una cuenca cercana (Cuenca del Río San Jorge), donde ocurre minería de ferroníquel, carbón y oro. Esta actividad se ha expandido en años recientes debido al incremento en la producción de ferroníquel, la construcción de una planta termoeléctrica a base de carbón (23) y la apertura de muchos frentes artesanales de minería de oro. Investigaciones recientes en esta área han mostrado altas concentraciones atmosféricas de $\mathrm{Hg}$ que son transportadas a otras áreas diferentes del área de explotación minera (24). Entonces, los altos niveles de $\mathrm{Hg}-\mathrm{T}$ en peces podrían reflejar el transporte atmosférico de áreas mineras cerca al embalse, o podría simplemente resultar de una mayor movilidad $\mathrm{de} \mathrm{Hg}$ en el área investigada.

En este sentido, los datos sobre parámetros de agua recolectados del embalse Urrá, mostraron un incremento de las condiciones anóxicas, acidificación y productividad con base en la 
in recent years due to an increase in the ferronickel production, the building of the new coal-based thermoelectric plant (23) and the opening of many artisanal fronts of gold mining. Recent research in this area has shown high atmospheric $\mathrm{Hg}$ concentrations that are transported to other areas outside the mining areas (24). Thus, the high $\mathrm{T}-\mathrm{Hg}$ levels in fish could reflect atmospheric transport from mining areas near the reservoir, or could simply result from a higher mobility of $\mathrm{Hg}$ in the area investigated.

In this regard, the data about to water parameters collected from the Urrá reservoir, showed an increase in the anoxic conditions, acidification and productivity based on OM concentration (Figure 3); conditions that enhance the rates of $\mathrm{Hg}$ methylation (8) and subsequent biomagnification in food chain. Indeed, it has been reported that acidic $\mathrm{pH}$ values increase mercury uptake by freshwater unicellular algae, primary producers in aquatic food chains (25). In the same direction, are highlighted the significant $(p<0.05)$ correlations recorded in this study between $\mathrm{T}-\mathrm{Hg}$ concentrations of $\mathrm{C}$. kraussii with $\mathrm{OM}, \mathrm{pH}$ and DO values of water (Table 2).

On the other hand, the $\mathrm{T}-\mathrm{Hg}$ concentration in water $(0.14 \pm 0.04 \mu \mathrm{g} / \mathrm{L})$ measured in 2009 in the Urrá reservoir was higher than the background values $(0.003 \mu \mathrm{g} / \mathrm{L})$ reported for Lago Manso reservoir in Brazil (6). Most likely, this difference results from local sources of $\mathrm{Hg}$ contamination. This result could reflect atmospheric transport from gold mining areas not far from the reservoir, or it could simply result from higher mobility of $\mathrm{Hg}$ in the areas investigated as was describe above. However, the results obtained were lower than those reported in marshes or rivers of heavily contaminated areas, such as the Grande marsh in Colombia (average $0.33 \pm 0.03 \mu \mathrm{g} / \mathrm{L}$ ) (26) and rivers from Indonesia (up to $0.25 \mu \mathrm{g} / \mathrm{L}$ ) (27), which are located around gold mining areas.

The $\mathrm{Hg}$ concentrations in the sediment samples collected in this study $(173.2 \pm 14.1 \mu \mathrm{g} / \mathrm{kg} \mathrm{dw})$ were lower than the values of $\mathrm{Hg}$ in sediment for other contaminated areas in Colombia, such as Ayapel marsh (243 $\pm 62 \mu \mathrm{g} / \mathrm{kg} \mathrm{dw}$ ) (28) and marshes of Mina Santa Cruz (up to $355 \mu \mathrm{g} / \mathrm{kg} \mathrm{dw}$ ) (29), but greater than those reported in areas such as the marshes from Caimito village in the San Jorge River basin, Colombia $(155 \pm 16 \mathrm{ug} / \mathrm{kg} \mathrm{dw})(11)$, which are also impacted by gold mining.

According to the above description, The $\mathrm{Hg}$ concentrations in the waters and favorable physicochemical characteristics present in the Urrá reservoir, may produce a greater efficiency of metal uptake by the fish and cause higher concentrations compared with other ecosystems. concentración de MO (Figura 3); condiciones que aumentan las tasas de metilación de $\mathrm{Hg}$ (8) y la subsiguiente biomagnificación en la cadena alimenticia. De hecho, se ha reportado que valores ácidos de $\mathrm{pH}$ incrementan la captación de $\mathrm{Hg}$ por algas unicelulares de agua dulce, principales productores en la cadenas alimenticias acuáticas (25). En el mismo sentido, se resaltan las correlaciones significativas $(p<0.05)$ registradas en este estudio entre las concentraciones de $\mathrm{Hg}-\mathrm{T}$ de $\mathrm{C}$. kraussii con los valores de $\mathrm{MO}, \mathrm{pH}$ y OD del agua (Tabla 2).

Por otro lado, la concentración de $\mathrm{Hg}-\mathrm{T}$ en agua $(0.14 \pm 0.04 \mu \mathrm{g} / \mathrm{L})$ medida en 2009 en el embalse Urrá fue mayor que los valores de base (background) $(0.003 \mu \mathrm{g} / \mathrm{L})$ reportados para el embalse Lago Manso en Brasil (6). Posiblemente, esta diferencia resulta de fuentes locales de contaminación de $\mathrm{Hg}$. Este resultado podría reflejar el transporte atmosférico de áreas de minería de oro no lejos del embalse, o podría simplemente resultar de una mayor movilidad de $\mathrm{Hg}$ en las áreas investigadas como se describió anteriormente. Sin embargo, los resultados obtenidos fueron menores que los reportados en ciénagas o ríos de áreas altamente contaminadas, tales como la Ciénaga Grande en Colombia (promedio $0.33 \pm 0.03 \mu \mathrm{g} / \mathrm{L}$ ) (26) y ríos de Indonesia (hasta $0.25 \mu \mathrm{g} / \mathrm{L}$ ) (27), ubicados alrededor de áreas de minería de oro.

Las concentraciones de $\mathrm{Hg}$ en las muestras de sedimentos recolectadas en este estudio $(173.2 \pm 13.1 \mu \mathrm{g} / \mathrm{kg} \mathrm{ps})$ fueron menores que los valores de $\mathrm{Hg}$ en sedimentos de otras áreas contaminadas en Colombia, como la Ciénaga de Ayapel (243 $\pm 62 \mu \mathrm{g} / \mathrm{kg}$ ps) (28) y las ciénagas de mina Santa Cruz (hasta $335 \mu \mathrm{g} / \mathrm{kg}$ ps) (29), pero mayores que las reportadas en áreas como las ciénagas del poblado El Caimito en la cuenca del Río San Jorge, Colombia (155 $\pm 16 \mu \mathrm{g} / \mathrm{kg}$ ps) (11), que también son impactadas por la minería de oro.

De acuerdo con lo antes descrito, las concentraciones de $\mathrm{Hg}$ en el agua y las características fisicoquímicas favorables presentes en el embalse Urrá, pueden producir una mayor eficiencia de captación del metal por los peces y causar mayores concentraciones comparadas con otros ecosistemas.

En conclusión, las concentraciones de $\mathrm{Hg}$-T en las especies de peces evaluadas en el embalse Urrá incrementaron después del represamiento. Las variables de calidad de agua mostraron condiciones que favorecen la metilación del $\mathrm{Hg}$ y su biomagnificación, esta última fue evidente en la cadena alimenticia de los peces del embalse, lo 
In conclusions, the $\mathrm{T}-\mathrm{Hg}$ concentrations in the evaluated fish species of the Urrá reservoir increased after impoundment. The water quality variables showed conditions favoring the $\mathrm{Hg}$ methylation and its biomagnification, this last was evident in the fish food chain of the reservoir, which poses a risk for riverside population that consume these fish. This study illustrates one of the potentially negative consequences of the construction of hydroelectric reservoirs and shows the need to include these potential environmental costs in the assessment of the impact of future projects of this type.

\section{Acknowledgments}

The authors thank the University of Cordoba, Montería (Colombia), for financial support (Grant FCB-032007), and the fishermen from Urrá reservoir for their help in catching fish. cual supone un riesgo para la población ribereña que consume estos peces. Este estudio ilustra una de las potenciales consecuencias negativas de la construcción de embalses hidroeléctricos y muestra la necesidad de incluir estos potenciales costos ambientales en la evaluación del impacto de futuros proyectos de este tipo.

\section{Agradecimientos}

Los autores agradecen a la Universidad de Córdoba, Montería (Colombia), por su apoyo financiero (Aportes FCB-03-2007), y a los pescadores del embalse de Urrá por su colaboración con la captura de los peces.

\section{REFERENCES}

1. Guzzi G, La Porta C. Molecular mechanisms triggered by mercury. Toxicol 2008; 244(1): 1-12.

2. United Nations Environment Programme. Global mercury assessment 2013. Sources, emissions, releases and environmental transport. Geneva: UNEP Chemicals Branch; 2013. (En línea, accesado 5 Mayo 2014). URL disponible en: http://www.unep.org/PDF/PressReleases / GlobalMercuryAssessment2013.pdf.

3. Pottinger L. World rivers review. [Online] International rivers 2010; 25(2):1-16. URL available in: http://www.internationalrivers.org/ files/attached-files/wrr_june_2010.pdf

4. St. Louis VL, Rudd JWM, Kelly CA, Bodaly RA, Paterson MJ, Beaty KG et al. The rise and fall of mercury methylation in an experimental reservoir. Environ Sci Technol 2004; 38(1): 1348-1358.

5. Hylander L, Gröhn J, Tropp M, Vikström A, Wolpher H, De Castro e Silva E. Fish mercury increase in Lago Manso, a new hydroelectric reservoir in tropical Brazil. J Environ Manage 2006; 81(2): 155-166.

6. Tuomola L, Niklasson T, de Castro e Silva E, Hylander L. Fish mercury development in relation to abiotic characteristics and carbon sources in a six-year-old, Brazilian reservoir. Sci Total Environ 2008; 390(1): 177-187.
7. Hall B, Cherewyk K, Paterson M, Bodaly R. Changes in methyl mercury concentrations in zooplankton from four experimental reservoirs with differing amounts of carbon in the flooded catchments. Can J Fish Aquat Sci 2009; 66(11): 1910-1919.

8. Ullrich S, Tanton T, Abdrashitova S. Mercury in the aquatic environment: A review of factors affecting methylation. Crit Rev Environ Sci Technol 2001; 31(3):241-293.

9. Marrugo-Negrete J, Olivero J, Lans E, Benitez L. Total mercury and methylmercury concentrations in fish from the Mojana region of Colombia. Environ Geochem Health 2008a; 30(1): 21-30.

10. Marrugo J, Lans E, Benítez L. Hallazgo de mercurio en peces de la Ciénaga de Ayapel, Córdoba, Colombia. Rev MVZ Córdoba 2007; 12(1):878-886.

11. Olivero-Verbel J, Johnson-Restrepo B, MendozaMarín C, Paz-Martinez R, Olivero-Verbel R. Mercury in the aquatic environment of the Village of Caimito at the Mojana Region, North of Colombia. Water Air Soil Pollut 2004; 159(1): 409-420.

12. Unidad de Planeación Minero Energética UPME. Reporte mensual de generación hidroeléctrica por embalse. Enero 2014. UPM; 2014. (En línea, accesado 25 Septiembre 2014). URL disponible en: http://www.upme.gov.co/Reports/Default. aspx?ReportPath=\%2fSIEL+UPME\%2fGenera ci\%C3\%B3n\%2fEmbalses+(SIN). 
13. Unidad de Planeación Minero Energética UPME. Plan de expansión de referencia generación - transmisión 2010-2024. Bogotá DC.: UPM; 2010a. (En línea, accesado 16 Octubre 2011). URL disponible en: http://www.upme. gov.co/Docs/Plan_Expansion/2010/Plan_ Expansion_2010-2024_Definitivo.pdf.

14. Marrugo-Negrete JL, Ruiz-Guzmán JA, Díez S. Relationship between mercury levels in hair and fish consumption in a population living near a hydroelectric tropical dam. Biol Trace Elem Res 2013; 151(2): 187-194.

15. Ruiz-Guzmán JA, Marrugo-Negrete JL, Díez S. Human exposure to mercury through fish consumption: Risk assessment of riverside inhabitants of the Urrá reservoir, Colombia. Hum Ecol Risk Assess 2014; 20(5): 1151 - 1163.

16. Valderrama M, Salas F, Solano D. Los peces y las pesquerías en el embalse de Urrá 2001 - 2005. Montería: Fundación bosques y humedales / Empresa Urrá S.A. E.S.P; 2006.

17. Ospina J, Gay C, Conde A, Magaña V, SánchezTorres $\mathrm{G}$. Vulnerability of water resources in the face of potential climate change: generation of hydroelectric power in Colombia. Atmósfera 2009; 22(3): 229-252.

18. United States Environmental protection Agency. Methods 2451 for determination of mercury in water. Cincinnati, OH: USEPA; 1994. (En línea, accesado 12 Agosto 2003). URL disponible en: http://water.epa.gov/scitech/methods/cwa/ bioindicators/upload/2007_07_10_methods_ method_245_1.pdf.

19. United States Environmental Protection Agency. Method 7471B for determination of mercury in solid or semisolid waste. Cincinnati, $\mathrm{OH}$ : USEPA; 1998. (En línea, accesado 12 Agosto 2003). URL disponible en: http://www.epa.gov/osw/hazard/ testmethods/sw846/pdfs/7471b.pdf.

20. American Public Health Association - American Water Works Association - Water Environment Federation. Standard methods for the examination of water and wastewater. 21th Ed. New York: APHA-AWWA-WEF-APHA; 2005.

21. FishBase World Wide Web: Search by species [en línea]. 2010. (fecha de acceso 14 de diciembre de 2010). URL disponible en: http://www. fishbase.org.
22. Food and Agriculture Organization / World Health Organization, Joint FAO/WHO Food Standards Programme Codex Committee on Contaminants in Foods CCCF, Seventh Session. Discussion paper on the review of the guideline levels for methylmercury in fish and predatory fish. CX/CF 13/7/16. Moscow: FAO/WHO; 2013. (En línea, accesado 9 Diciembre 20014). URL disponible en: $\mathrm{ftp}: / / \mathrm{ftp}$.fao.org/codex/meetings/cccf/cccf7/ cf07_16e.pdf.

23. Departamento Nacional de Planeación DNP. Documento 3565 del Conpes (Consejo Nacional de Política Económica y Social). Importancia estratégica del proyecto de generación termoeléctrico a carbón GECELCA 3. Bogotá DC.: DNP; 2009. (en línea, accesado 18 enero 2010). URL disponible en: https://pwh. dnp.gov.co/Portals/0/archivos/ documentos/ Subdireccion/Conpes/3565.pdf.

24. Marrugo-Negrete JL, Urango-Cardenas I, Burgos S, Díez S. Atmospheric deposition of heavy metals in the mining area of the San Jorge river basin, Colombia. Air Qual Atmos Health 2014; 7:577 -588. doi: 10.1007/s11869-014-0260-0.

25. Le Faucheur S, Tremblay Y, Fortin C, Campbell PGC. Acidification increases mercury uptake by a freshwater alga, Chlamydomonas reinhardtii. Environ Chem 2011; 8(6): 612-622.

26. Marrugo-Negrete J, Benitez L, Olivero J. Distribution of mercury in several environmental compartments in an aquatic ecosystem impacted by gold mining in Northern Colombia. Arch Environ Contam Toxicol 2008b; 55(2): 305-316.

27. Limbong D, Kumampung J, Ayhuan D, Arai T, Miyazaki N. Mercury pollution related to artisanal gold mining in north Sulawesi Island, Indonesia. Bull Environ Contam Toxicol 2005; 75(5): 989 $-996$.

28. Marrugo-Negrete J, Benítez L, Olivero-Verbel J, Lans E, Vazquez F. Spatial and seasonal mercury distribution in the Ayapel Marsh, Mojana region, Colombia. Int J Environ Health Res 2010; 20(6):451-459.

29. Olivero J, Solano B. Mercury in environmental samples from a waterbody contaminated by gold mining in Colombia, South America. Sci Total Environ 1998; 217(1-2): 83 - 89. 